\title{
Black Women's Preferences for Embedding Mental Health Services in An Obstetrics Setting
}

\author{
Courtney Glickman'), Huynh-Nhu Le²), Hillary A. Robertson3), Ruthie Arbit3), \\ Aimee Danielson ${ }^{3)}$, Loral Patchen ${ }^{4)}$, Melissa Fries4), Rachel Scott5), Matthew G. Biel6) \\ 1)Dept of Counseling and Human Development, The George Washington University \\ 2)Dept of Psychology, The George Washington University \\ 3)Dept of Psychiatry, Georgetown University Medical Center \\ 4) MedStar Washington Hospital Center \\ 5)MedStar Health Research Institute and Washington Hospital Center \\ 6) Medstar Georgetown University Hospital, Georgetown University Medical Center
}

\section{ABSTRACT}

Background: Untreated perinatal depression is a significant public health issue that disproportionately affects low-income black women and may lead to higher maternal mortality rates and disparate birth outcomes. Despite the growing literature documenting prevalence and risk for perinatal depression among black women, our knowledge of patient preferences of mental health interventions in obstetrical (OB) settings among this population is limited. This study explored mental health treatment preferences among black pregnant and postpartum women in an urban OB practice, serving predominantly black women with public insurance.

Subjects and Method: We employed a qualitative approach to examine Black perinatal women's mental health preferences in an $\mathrm{OB}$ setting. The study sample consisted of 14 perinatal women at an urban obstetrics practice in Washington, DC. Semi-structured interviews were conducted to gain insight into their emotional experiences during pregnancy and postpartum, and to obtain patient recommendations for mental health interventions within an obstetrics setting. Patient interviews were audio-recorded, transcribed, and analyzed by two coders using inductive thematic analysis and consensus procedures.

Results: Participants reported several general content themes: provider- patient relationship and intervention topic feedback. Within the overall content theme of provider-patient relationship, black women noted differences in their overall levels of comfort in discussing stress and mental health issues. Subthemes of authentic connection and perceived stigma emerged. Relevant subthemes on intervention topic preferences discussed content, modality, and logistics.

Conclusion: Our findings support the importance of offering integrative, culturally competent, and accessible mental health interventions in order to prevent and treat perinatal depression among low-income black women in $\mathrm{OB}$ settings.

Keywords: pregnancy, perinatal, depression, black women

\section{Correspondence:}

Courtney Glickman. Dept of Counseling and Human Development, The George Washington University2134 G St NW, Washington, DC 20052. Email: Cglickman22@gmail.com.

Cite this as:

Glickman C, Le HN, Robertson HA, Arbit R, Danielson A, Patchen L, Fries M, Scott R, Biel MG (2020). Black Women's Preferences for Embedding Mental Health Services in An Obstetrics Setting. J Matern Child Health. 05(04): 352-364. https://doi.org/10.26911/thejmch.2020.05.04.02.

Journal of Maternal and Child Health is licensed under a Creative Commons Attribution-NonCommercial-ShareAlike 4.0 International License.

\section{BACKGROUND}

Depression in the perinatal period (from pregnancy through the first postpartum year) is highly prevalent and carries adverse consequences for mothers, infants, and 
families. Although one in seven women suffer from perinatal depression, the risks of perinatal depression and the severity of its consequences are not evenly distributed in the population (Gavin et al., 2005; Goodman et al., 2013). Mothers from lowsocioeconomic backgrounds, who are disproportionatelywomen of color, typically report higher levels of depressive symptoms than white women (Beeghly et al., 2003; Howell et al., 2005). Black women in the United States may be particularly impacted by increased levels of economic hardship. Black women experience higher rates of psychological stressors such as trauma that increase their risk of perinatal mental health disorders (Seng et al., 2011). Additional layers of vulnerability, such as exposure to racial discrimination, access to fewer resources, and systemic inequities, often intersect to create complex mental health treatment needs among low-income black women, making it essential to address these factors in the context of perinatal care in order to forestall negative outcomes for both mothers and their children.

Despite this greater need for support around perinatal mental health, rates of screening and treatment are lower among black women than white women, and this disparity is particularly prominent among women of lower socioeconomic status (Geier et al., 2015; Lara-Cinisomo et al., 2009). In addition, low-income black women have lower rates of initiating and continuing in mental health care than white women, likely as a result of numerous barriers at the systems, provider, and individual levels (Kozhimannil et al., 2011). While the majority of obstetrical providers acknowledgea responsibility in recognizing perinatal depression, there are significant provider level barriers to screen and provide follow-up interventions, including the absence of systematic referral pro- cesses, time constraints, limited knowledge of available resources, limitations in professional training to conduct screenings, lack of comfort with managing depression, and a perceived reluctance of their patients to engage in mental health treatment (Byatt et al., 2012). In addition, black women report less satisfaction with healthcare, have less trust in their providers, and perceive less respect and acceptance from their providers, as compared withwhite women (Armstrong et al., 2008). Further, there is a need for providers tounderstand women's depression through the social context in which they live,since many of the determinants of health disparities seen among lowincome black women with depressive symptoms are social or contextual (Nicolaidis et al., 2010). Research has found that developing models of prenatal care congruent with the realities of black women's lives can contribute to the improvement of patientprovider relationships and potentially impact birth outcomes (Lori et al., 2011).

Untreated perinatal depression is a significant public health issue that disproportionately affects low-income black women and may lead to higher maternal mortality rates and disparate birth outcomes (Creanga et al., 2017; Peters et al., 2014). When left untreated, perinatal depression may contribute to compromised parenting and poor outcomes for children, including impaired social-emotional functioning, insecure attachment, and cognitive and developmental delays (Beeghly et al., 2003; Creanga et al., 2017). Despite the growing literature documenting prevalence and risk for perinatal depression among black women, our knowledge of patient preferences of mental health interventions in obstetrical (OB) settings among this population is limited. OB settings provide an optimal opportunity for identification, prevention, and treatment of perinatal 
depression, as women are more likely to access medical care during pregnancy (Flynn et al., 2006; Goodman et al., 2013; Marcus, et al., 2003). During the perinatal period, this venue is among the most accessible in health care andcould reach a larger proportion of women. The concept of embedding mental health care within the OB setting promotes a more holistic and integrated approach, strengthening detection and access to treatment and prevention services for perinatal depression.

Additional research examining barriers and facilitators to treatment utilization and intervention preferences of black women in the peripartum period is essential in order to design and implement effective prevention and treatment efforts specifically for this population. This study explored mental health treatment preferences among black pregnant and postpartum women in an urban OB practice, serving predominantly black women with publicinsurance. Specifically, we examined: 1) What are black women's preferences for quality provider-patient relationships? 2) What are black women's treatment preferences for perinatal depression?.

\section{SUBJECTS AND METHOD}

\section{Study Design}

This study was part of a larger investigation of pregnant and postpartum women's emotional experiences, mental health treatment and intervention preferences in the context of a feasibility study to integrate mental health services within an OB setting. We employed a qualitative approach to examine Black perinatal women's mental health preferences in an OB setting. Qualitative methods allow for in-depth, nuanced examination of patient preferences and give voice to participants who have been historically underrepresented or marginalized in health research (Woodley and Lockard, 2016).

\section{Participants and Setting}

We recruited participants from an urban OB practice that is part of a larger hospital setting serving predominantly publiclyinsured blackwomen in Washington, DC. All women who were pregnant or postpartum (up to one year after birth), 18 years of age or older, and receiving care at the OB practice in which recruitment took place were eligible to participate. The practice is part of a larger tertiary care center providing care for women with varying medical risk status. The hospital has 3,500-4,000 deliveries annually and 1,200 of these are from women served in this practice, where an average of 250 patients a week receive outpatient care. The majority of health providers (obstetricians, nurse practitioners, and midwives) and the social worker in this particular setting were white,indicating a low racial concordance between patients and providers.

\section{Study Instrument}

Participants completed a brief demographic questionnaire and two brief measures of depressive and anxiety symptoms prior to their interview.

The Edinburgh Postnatal Depression Scale (EPDS; Cox et al., 1987) is a 10-item measure that has been validated as a depression screening measure in both prenatal and postpartum samples (Adouard et al., 2005). The EPDS is increasingly used in public health and social service programs serving pregnant and postpartum women of racially, ethnically, and socioeconomically diverse backgrounds at risk for depression (Lee-King, 2012). Items are scored on a four-point Likert-type scale (o to 3). A higher score indicates higher frequency or severity of symptoms, with total scores ranging from o to 30 . Women with a score of 13 or greater are considered to have 
clinically significant levels of depression (Cox et al.,1996).

The Generalized Anxiety Disorder Scale (GAD-7) is a seven-item measure that assess the severity of generalized anxiety symptoms. A higher score reflects higher levels of anxiety. Items are scored on a four-point Likert-type scale (o to 3). A GAD-7 score 10 or greater is considered indicative of generalized anxiety disorder and scores in the 5-9 range are indicative of mild generalized anxiety (Spitzer et al., 2006).

\section{Procedure}

Participants were approached by a member of the study team in the waiting room or while waiting for the provider in the examination room, given information about the study, and asked if they wanted to participate. This team member, who is white and has a social work background, was concurrently beginning to work as a mental health therapist in the practice as part of a larger study. This present study is part of a larger study examining pregnant and postpartum women's emotional experiences and the feasibility of integrating mental health care in an OB setting. This study was approved by the institutional review board at the hospital and university.

Participants were given the option to meet with the study social worker after their appointment for the interview or to schedule time on a later date. Prior to each interview, potential participants were provided with information about the study and if they were interested in participating, they signed a written consent document and were provided a $\$ 30$ gift card.

Participants completed the two measurement scales and then completed semistructured interviews. Interviews were conducted in person to gain insight into patients' emotional experiences during pregnancy and postpartum, and to obtain patient recommendations for mental health interventions within an $\mathrm{OB}$ setting. The same interview guide was used with all participants. This guide included open-ended questions such as, "We are thinking about offering a program at this practice, free of charge, to help pregnant women and new moms manage stress and worries. In it, we would talk about common pregnancy problems and how to manage them. Please tell me what you would like in a program like this?" and "If you had stressors, worries or emotional difficulties, how comfortable would you feel talking about these issues with your provider?", allowing the interviewer to incorporate an inductive approach and participants to express preferences in their own words. Topics explored in the interview included women's experiencesin the OB setting, relationship and communication with providers, sources of support, and experiences with mental health. On average, each interview was37 minutes, ranging from 28 to 48 minutes. All interviews were audio recorded.

\section{Data Analysis}

Descriptive statistics were conducted inIBM SPSS Statistics (IBM Corp, 2017) for demographic information and measures of depression and anxiety. Patient interviews were transcribed and analyzed by two coders using inductive thematic analysis and consensus procedures (Braun and Clarke, 2006). First, two codersreviewed all transcripts and developed an initial codebook. Next, each coder independently coded each of the transcripts. After coding, the researchers met to discuss discrepancies and resolve disagreements.

\section{RESULTS}

Demographic characteristics of study participants are shown in Table 1. A total of 9 (64.3\%) patients who completed the interview were pregnant and 13 were black 
Glickman et al./ Black women's preferences for embedding mental health services

(92.9\%). The mean age was 27 years $(\mathrm{Mean}=27 ; \mathrm{SD}=3.56)$ and patients had an average of two children (Mean $=2.25, \mathrm{SD}=$ 1.17). No patients reported current mental health treatment, but some patients had previously received mental health treatment $(21.4 \%, \mathrm{n}=3)$.

All participants rated their health as "good" or better. Participants reported a mean score of $4.15(\mathrm{SD}=3.51)$ on the EPDS and a mean score of $3.08(\mathrm{SD}=2.90)$ on the
GAD-7, indicating low reported depressive and anxiety symptoms, respectively.

Participants described a range of personal experiences with perinatal mental health. Participant responses were reviewed and analyzed for each research question, yielding two general content themes related to the provider-patient relationship and treatment preferences (see Figure 1). Each theme is described below with exemplar quotes that illustrate the substance of material presented.

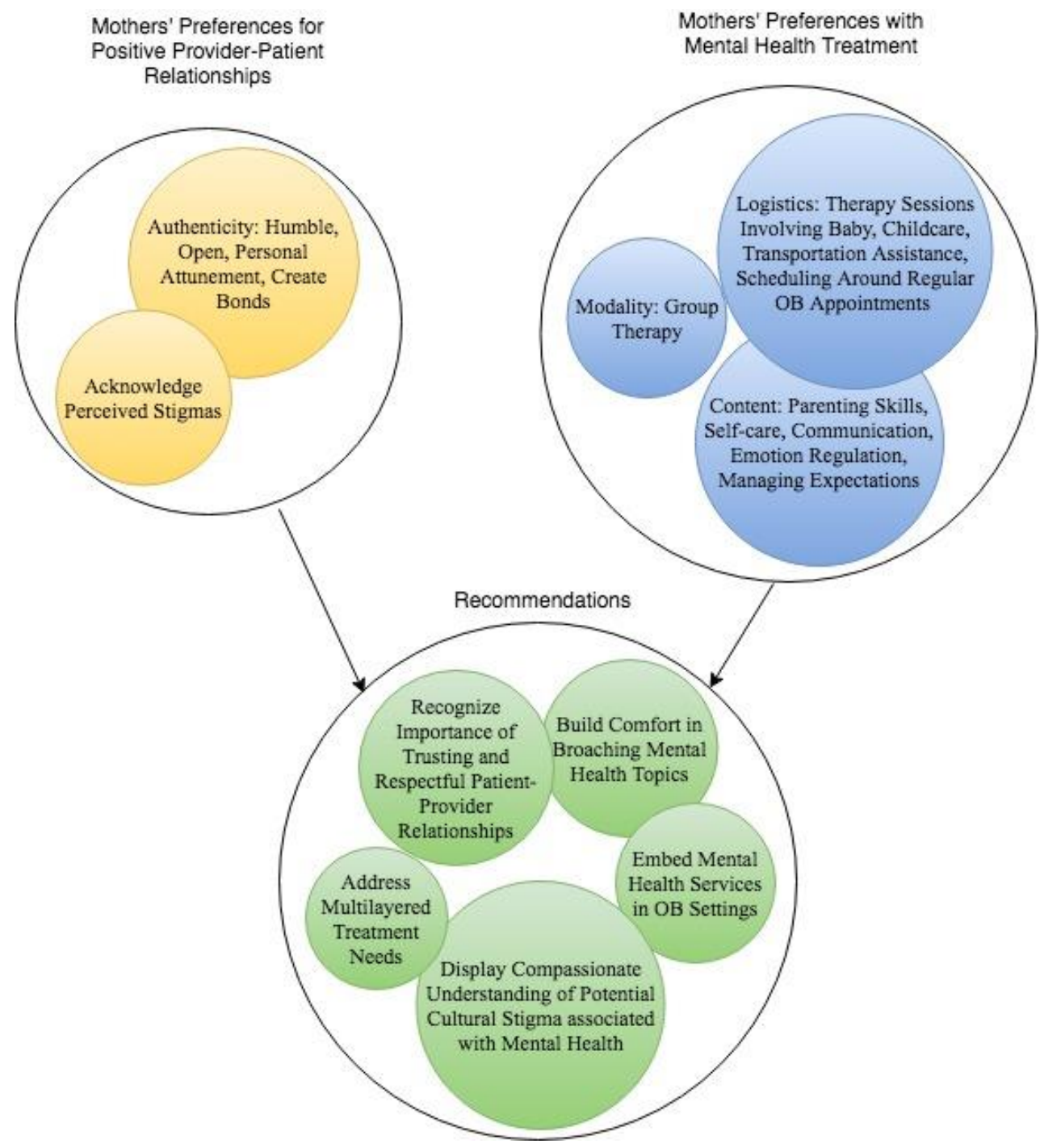

Figure 1. Themes and recommendations regarding the provider-patient relationship and treatment preferences in an $O B$ setting among Black perinatal women 
Provider Patient Relationship. When examining responses describing the provider-patient relationship, subthemes of authentic connection and perceived stigma emerged.Participants noted differences in their overall levels of comfort in discussing stress and mental health issues with their providers.

Authentic connection. There was a clear preference expressed in discussing mental health issues with a provider who is "humble" and "open". One postpartum participant stated:

You know, I really appreciate the kindness and the...the openness with the, some of the doctors that I've had, you know? You know, even like when they weren't sure about something, they were open enough to say, okay I'm not sure, I'm gonna go and get a colleague and you know so that made me feel comfortable that they are, they acknowledge that they don't have the answers to everything. You know, that they also need help, which is not a bad thing, you know?

Participants also indicated a desire for providers to build their comfort levels in broaching mental health topics and creating more of a "bond" with their patients. One postpartum participant with two children stated in regard to her long-term relationship with her provider who also delivered both of her children:

Yes and even still - I'm still not comfortable with her and talking with her about different things. I do feel like doctors should definitely build some relationships so that their patients could feel comfortable speaking on things. And if not, we leave the office with so many things on our mind that we couldn't even get out.

The authenticity of the provider-patient relationship directly related to barriers in receiving and following through with mental health referrals. Among those who received referrals, participants noted a lack of coordination of care or wrap around services. Some participants added that the specific referrals did not feel suited to their individual needs or lacked a more personal level of attunement. Attunement was conceptualized as meaning the provider was present with the patient in an interactive manner and accurately captured the sense of how they were feeling in the moment--in essence, providing the patient with a sense of feeling seen and heard. Participants who perceived their interactions with providers as lacking comprehensive care reported feeling as though the communication was dismissive rather than responsive and attentive to their emotional needs.One postpartum participant noted:

When I had mentioned something about how I was feeling she [provider] just automatically, she just referred me to the therapist. We didn't talk about it at all, she was just like "maybe you should see a therapist". I wouldn't say she didn't care, but she really didn't want to talk about it.

Some participants expanded on the concept of genuineness and relatability further to note that a more formal and less person-centered approach came across as insensitive. One postpartum participant describes her experiences stating:

And I'm like here not because you know, I'm here in a high-risk clinic not because I'm well, you know and everything's in control, but I'm having some additional problems and that's why I'm here so, the little comments you know, to me is unnecessary and it made me feel uncomfortable. You know, at the same time. But that is why I say, be more sensitive to you know, what's going on with that particular person 
versus making comment "eh, eh, why is your blood pressure high," Or like they're driving your anxiety level up here, you know.

The comments emphasized authentic patient-provider relationships with characteristics that indicate an approach that valued each patient individually. Many participants describednarratives that detailed frustration with being just a number or lacking in empathy. One postpartum participant reported:

I feel like they just get us in and out. Instead of, you know, talking to us they talk at us it's like a routine. They ask the same questions with every patient so it's a routine. And they are just looking for the answer to the question instead of listening and paying attention to us.

Perceived Stigma. Perceived stigma emerged as a frequent concern for participants, which was often associated with fears of repercussions or consequences from being "honest" about mental health issues or having a mental health diagnosis on their medical record. One postpartum participant stated:

I would be comfortable, but then I'm not sure how much I'd be able to say to someone without them assuming the worst or, those types of repercussions or consequences from just being honest and talking about my personal feelings and everything. Because it's tied to, like medical records and things like that. You don't want that to reflect badly on you later on.

In terms of communicating with providers about interpersonal or relational conflicts at home, participants reported being more apprehensive, noting these issues were "too personal," and they were more likely to open up to a main source of support (e.g., family, friends). In contrast, participants were more likely to discuss substance use issues with providers. When the interviewer queried whether the participants perceived substance use to be a physical health topic rather than strictly mental health, respondents' answers were affirmative.

Treatment Preferences. When asked about potential program development to support pregnant and postpartum women with staying healthy and coping with stress, participants noted three relevant subthemes of modality, content, and logistics.

Modality. Participants cited group therapy as favorable over individual. Narratives related to social support and shared experiences as a positive aspect of group therapy were frequent. For example, one pregnant participant stated:

It's very good for pregnant women because...anything is good for pregnant women. Because at some points you just feel like you're not like you used to be and everything changes, the way you walk, the way you wake up...so if there are good quality times that you can share even in...it's a good thing. It's very good for the baby too because if their mother is happy and she feels like she has help or support, that's good.

Another postpartum participant with two children expressed a preference for groups noting the importance of leaving the home and remaining connected to sources of support:

I think a group would be better just for the social interaction, just to be social. Cause when you have, you first have a baby you can't really hang out or really do much. So I think that would help the social aspect, that would help. Cause you feel like you're getting out but not really hanging out where you feel like you're not at home all the time. 
Content. Topics related to managing expectations, parenting skills (e.g., childcare, sleep, and nutrition), self-care, and tools for effective communication and emotion regulation were preferred. One postpartum participant stated:

Definitely dealing with the baby at different stages of their life. Also, stress management. Like, when you're almost at that boiling point where you just can't deal with the baby anymore, you know, back to the stress thing. Definitely for first time parents, you know, bathing the baby, changing diapers, all that kind of stuff.

Logistics. The respondents perceived various logistical barriers preventing women from taking advantage of interventions as well as potential facilitators to enhance the likelihood of treatment adherence. For example, nearly all participants noted childcare as a barrier to treatment. One postpartum participant stated:

Maybe if there's a situation where like, you can bring your children with you? Like if it's a family-oriented type of thing. Or if they have things for the children to do or children to play.

Several women reported transportation barriers. Many did not drive or could not afford parking and relied on the practice's location being close to a metro or bus station. Multiple participants recommended transportation options be provided by the practice. This is detailed in one pregnant participant's response:

If they could have a van and they could pick the moms up that's be... everybody doesn't have reliable transportation so maybe that'll be a reason why some moms may not show up because they don't want to tow their stroller and baby bags and all those things on public transportation.

Recommendations to schedule mental health appointments before or after OB appointments were also discussed to decrease difficulties with childcare and transportation.

\section{DISCUSSION}

The present study provides a preliminary investigation into the patient preferences of mental health interventions in OB settings among low-incomeblack women. Our study is an initial attempt to fill the gap on the unique experiences of black pregnant and postpartum women and supports efforts to understand perinatal mental health preferences as an essential component to the development and implementation of intervention programs. Overall, our findings align with the broader literature regarding black individuals' mental health preferences, acknowledging that cultural issues (e.g., stigma, importance of a trusting relationship with provider) and logistical issues (e.g., childcare and transportation) contribute to barriers in accessing and utilizing care (Alvidrez, 1999, Nadeem et al., 2008; Nicolaidis et al., 2010).

In regard to provider-patient relationships (see Figure 1), these women valued having trustworthy relationships with their providers as a means towards building individualized care and connections. Prior research has emphasized that many black women view the health care system as racially biased, noting past negative experiences attributed to racism, such as providers not spending enough time with them, not respecting their intelligence, not providing adequate explanations, and breaking their trust (Nicolaidis et al., 2010). In addition, our study found that perceived stigma from disclosure of mental health issues was associated with fears of repercussions or consequences. Heightened community and self-stigma as well as mistrust of the mental health profession have been attributed to psychological barriers in 
mental health treatment seeking behaviors among black people in the United States (Alvidrez, 1999; Nadeem et al., 2008; Nicolaidis et al., 2010).

Interestingly, findings were suggestive of participants perceiving substance use as aligning more with the domain of physical health than strictly mental health. As observed in other studies, this may be related to the self-stigma and the tendency for people to identify causal factors of illness that reflect the specific circumstances of their own lives (Hunt et al., 1989). Our findings support previous strategies for addressing barriers to the detection and treatment of depression in the black population including: 1) assessing for stigma toward mental health problems;2) making the connection between physical and mental health; 3) inquiring about patients' experience of life stressors, and social conflicts; 4)maintaining a respectful, open dialogue to understand an individual's style of coping with depressive symptoms have been recommended for addressing barriers to the detection and treatment of depression in the black population (Das et al., 2006).

Women are more likely to seek medical care during pregnancy, and women with multiple children tend to deliver in the same setting. Thus, OB settings are among the most accessible in health care during the perinatal period (Flynn et al., 2006; Goodman et al., 2013; Marcus, et al., 2003). These OB visits afford opportunities to connect with patients and allow the development of a quality provider-patient relationship. Recognizing perceived barriers and facilitating treatment have been shown to enhance treatment seeking (Byatt et al., 2012; Ward et al., 2013; Stevens et al., 2018). Thus, mental health services might be more likely to be accessed by low-income black women if mental health care is embedded within a patient's OB visit.

Data on treatment preferences (see Figure 1)revealed additional perceived barriers to participating in mental health treatment, particularly childcare needs and transportation barriers. Within this study, the shared experiencesof becoming a mother and the responsibilities of having a newborn were stressed as providing a level of communal safety. As the life transition of having a child felt isolating for some women, group modality was identified as preferable over individual modality. Integrating mental health care with options for group therapy within the OB setting could provide an optimal opportunity for identification and treatment of perinatal depression as well as a supportive and destigmatizing space.

Research on treatment for depression in general adult populations has found that attention to patient preferences is associated with patients being more likely to enter treatment, to receive efficacious treatments, andto show an overall improvement in depression symptom levels (Clever et al., 2006; Lin et al., 2005). Adding to this literature, we sought information that would guide the design of future interventions that pregnant and postpartum lowincomeblack women would find affirming and accessible. Participants strongly preferred intervention topics focused on managing expectations, parenting skills (e.g., childcare, sleep, and nutrition), self-care, and tools for effective communication and emotion regulation. Based on our findings, patients value the importance of a trusting and respectful patient-provider relationship, are sensitive to compassionate understanding of potential cultural stigma associated with mental health treatment, and value the importance of addressing complex factors, logistical or otherwise, that 
intersect to create multilayered treatment needs among low-incomeblack women.

This study's limitations are its small sample size and narrow geographic scope, which may decrease the generalizability of the findings. However, the patients' perspectives may be similar to other black women in urban communities. Although the sample size of this study $(\mathrm{N}=14)$ was appropriate for the qualitative analyses completed, a larger sample would increase the study's external validity. In addition, we were unable to identify differences between pregnant and postpartum women. Moreover, there is also a lack of generalizability inherent in the nature of qualitative research. As with any study based upon selfreport data, there is potential for bias.

Another limitation to this study was the lack of racial concordance between participants and interviewer. It is unclear the extent to which this hindered opportunities for a more in-depth explorations of experiences or perceptions of discrimination as it relates to mental health interventions. Nevertheless, given that the interviewer was concurrently working as staff within this setting,there may have been a level of familiarity. In addition, as the majority of health providers in this setting were white, this racial disparity is not unusual. Further research is warranted to examine the relationship between discrimination, providerpatient relationship, and mental health integration in OB settings among black women.

Perinatal depression among lowincome black women is a significant public health problem. Our findings have a number of implications for health care practice, policy, and practitioners' education in OB settings. Factors including patient mental health preferences, barriers, and facilitators to treatment must be considered to inform improvements to health care access and interventions. Consistent with previous literature, these findings illuminate the need for collaborations among researchers, health care providers, and the patients served to develop programs aimed at providing mental health interventions for pregnant and postpartum black women.

In addition, providers have an obligation to mitigate health disparities among racial and ethnic minorities. We hope to highlight the importance of education and strategies to strengthen cultural competence. Providing equitable and responsive mental health interventions in OB settings may help reduce disparities and improve outcomes. Further research should examine the efficacy of treatment interventions and reduction of barriers among pregnant and postpartum low-income black women. Our findings support the importance of offering integrative, culturally competent, and accessible mental health interventions in order to prevent and treat perinatal depression among low-income black women.

\section{AUTHOR CONTRIBUTION}

All authors whose names are listed certify that they have no affiliations with or involvement in any organization orentity with any financial interest or non-financial interest in the subject matter or materials discussed in this manuscript.

\section{CONFLICT OF INTEREST}

There are no conflicts of interest.

\section{FUNDING AND SPONSORSHIP}

This study was supported by funding from the Early Childhood Innovation Network and the J. Willard and Alice S. Marriott Foundation.

\section{ACKNOWLEDGEMENT}

The authors thank the participants and staff at the OB hospital setting. 
Glickman et al./ Black women's preferences for embedding mental health services

\section{REFERENCE}

Adouard F, Glangeaud-Freudenthal NMC, Golse B (2005). Validation of the Edinburgh postnatal depression scale (EPDS) in a sample of women with high-risk pregnancies in France. Archives of Women's Mental Health. 8(2): 89-95. https://doi.org/10.1007/s00737-005-0077-9.

Alvidrez J (1999). Ethnic variations in mental health attitudes and service use among low-income African American, Latina, and European American young women. Community Mental Health J. 35(6): 515-530. https://psycnet.apa.org/doi/10.1023/A:10187 59201290.

Armstrong K, McMurphy S, Dean LT, Micco E, Putt M, Halbert CH, Schwartz JS, Sankar P, Pyeritz RE, Bernhardt B, Shea JA (2008). Differences in the patterns of health care system distrust between blacks and whites. Journal of General Internal Medicine. 23(6): 827-833. https://doi.org/10.1007/s11606-008-0561-9

Beeghly M, Olson KL, Weinberg MK, Pierre SC, Downey N, Tronick EZ (2003). Prevalence, stability, and socio-demographic correlates of depressive symptoms in black mothers during the first 18 months postpartum. Matern Child Health J. 7(3): 157-168. https://doi.org/10.1023/a:1025132320321.

Braun V, Clarke V (2006). Using thematic analysis in psychology. Qualitative Research in Psychology. 3(2): 77-101. https://doi.org/10.1191/1478088706q po630a.

Byatt N, Simas T, Lundquist R, Johnson J, Ziedonis D (2012). Strategies for improving perinatal depression treatment in North American outpatient obstetric settings. Journal of Psychosomatic Obstetrics \& Gynecology.
33(4): 143-161. https://doi.org/10.3109/0167482x.2012.728649.

Clever SL, Ford DE, Rubenstein LV, Rost KM, Meredith LS, Sherbourne CD, Wang NY, Arbelaez JJ, Cooper LA (2006). Primary care patients' involvement in decision-making is associated with improvement in depression. Medical Care. 44(5): 398-405. https://doi.org/10.1097/o1.mlr.000o 208117.15531.da.

Cox JL, Chapman G, Murray D, Jones P (1996). Validation of the Edinburgh postnatal depression scale (EPDS) in non-postnatal women. Journal of Affective Disorders. 39(3): 185-189. https://doi.org/10.1016/0165-0327(96)oooo8-o.

Cox JL, Holden JM, Sagovsky R (1987). Detection of postnatal depression. Development of the 10-item Edinburgh postnatal depression scale. Br J Psychiatry. 150: 782-786. https://doi.org/10.1192/bjp.150.6.782.

Creanga AA, Syverson C, Seed K, Callaghan WM (2017). Pregnancy-related mortality in the United States, 2011-2013. Obstetrics \& Gynecology. 130(2): 366-373. https://doi.org/10.1097/aog.0000000000002114.

Das AK, Olfson M, McCurtis HL, Weissman MM (2006). Depression in African Americans: breaking barriers to detection and treatment. J Fam Pract. 55(1): 30-39. https://pubmed.ncbi.nlm.nih.gov/16388764/.

Flynn HA, Blow FC, Marcus SM (2006). Rates and predictors of depression treatment among pregnant women in hospital-affiliated obstetrics practices. General Hospital Psychiatry. 28(4): 289-295. https://doi.org/10.1016/j.genhosppsych.2006.04.002

Gavin NI , BN Gaynes, KN Lohr, MeltzerBrody S, Gartlehner G, Swinson T 
Glickman et al./ Black women's preferences for embedding mental health services

(2005). Perinatal depression: A systematic review of prevalence and incidence. Obstet Gynecol. 106 (5, Part 1): 1071-1083. https://doi.org/10.1097/01.aog.0ooo183597.31630.db.

Geier ML, Hills N, Gonzales M, Tum K, Finley PR (2015). Detection and treatment rates for perinatal depression in a state Medicaid population. CNS spectrums. 20(1): 11-19. https://doi.org/10.1017/s1092852914000510.

Goodman SH, Sona Dimidjian S, Williams KG (2013). Pregnant African American women's attitudes toward perinatal depression prevention. Cultural Diversity and Ethnic Minority Psychology. 19(1): 50-57. https://doi.org/10.1037/a0030565.

Howell EA, Mora PA, Horowitz CR, Leventhal $\mathrm{H}$ (2005). Racial and ethnic differences in factors associated with early postpartum depressive symptoms. Obstetrics \& Gynecology. 105 (6): 1442-1450. https://dx.doi.org/10.1097\%2Fo1.AOG.0000164050.341 26.37.

Hunt LM, Jordan B, Irwin S (1989). Views of what's wrong: diagnosis and patients' concepts of illness. Social Science and Medicine. 28(1989): 945956. https://doi.org/10.1016/0277-9536(89)90324-9.

Kozhimannil KB, Trinacty CM, Busch AB, Huskamp HA, Adams AS (2011). Racial and ethnic disparities in postpartum depression care among lowincome women. Psychiatric Services. 62(6): 619-625. https://dx.doi.org/10.1176\%2Fappi.ps.62.6.619

Lara-Cinisomo S, Griffin BA, Daugherty L (2009). Disparities in detection and treatment history among mothers with major depression in Los Angeles. Women's Health Issues. 19(4): 232-
242. https://doi.org/10.1016/j.whi.2009.03.007

Lee-King PAL (2012). Replicability of structural models of the Edinburgh Postnatal Depression Scale (EPDS) in a community sample of postpartum African American women with low socioeconomic status. Archives of Women's Mental Health. 15(2): 7786. https://doi.org/10.1007/soo737012-0260-8.

Lin P, Campbell DG, Chaney EF, Liu CF, Heagerty P, Felker BL, Hedrick SC (2005). The influence of patient preference on depression treatment in primary care. Annals of Behavioral Medicine. 30(2): 164-173. https://doi.org/10.1207/s15324796abm3002 $-9$.

Lori JR, Yi CH, Martyn KK (2011). Provider characteristics desired by African American women in prenatal care. Journal of Transcultural Nursing. 22 (1): 71-76. https://doi.org/10.1177/1043659610387149.

Marcus SM, Flynn HA, Blow FC, Barry KL (2004). Depressive symptoms among pregnant women screened in obstetrics settings. J women's health. 12(4): 373-380. https://doi.org/10.1089/154099903765448880.

Nadeem, E Lange JM, Miranda J (2008). Mental health care preferences among low-income and minority women. Archives of Women's Mental Health. 11(2): 93-102. https://dx.doi.org/10.1007\%2Fs00737-008-0002-0.

Nicolaidis C, Timmons V, Thomas MJ, Waters AS, Wahab S, Mejia A, Mitchell SR (2010) 'You don't go tell White people nothing': African American women's perspectives on the influence of violence and race on depression and depression care. Am J Public Health. 100(8): 1470-1476. 
Glickman et al./ Black women's preferences for embedding mental health services

https://dx.doi.org/10.2105\%2FAJPH. 2009.161950.

Peters RM, Benkert R, Templin TN, Cassidy-Bushrow AE (2014). Measuring African American women's trust in provider during pregnancy. Research in Nursing \& Health. 37(2): 144-154. https://doi.org/10.1002/nur.21581.

Seng JS, Kohn-Wood LP, McPherson MD, Sperlich M (2011). Disparity in posttraumatic stress disorder diagnosis among African American pregnant women. Archives of Women's Mental Health. 14(4): 295-306. https://dx.doi.org/10.1007\%2Fso0737-011-0218-2.

Spitzer RL, Kroenke K, Williams JBW, Löwe B (2006). A brief measure for assessing generalized anxiety disorder: The GAD-7. Archives of Internal Medicine. 166 (10): 1092-1097. doi:10.1001/archinte.166.10.1092.
Stevens NR, Heath NM, Lillis TA, McMinn K, Tirone V, Sha'ini M (2018). Examining the effectiveness of a coordinated perinatal mental health care model using an intersectional-feminist perspective. Journal of Behavioral Medicine. 41(5): 627-640. https://doi.org/10.1007/s10865-018-9973-0.

Ward TCS, Mazul M, Ngui EM, Bridgewater FD, Harley AE (2013). 'You learn to go last': perceptions of prenatal care experiences among African-American women with limited incomes. Matern child health J. 17(10): 1753-1759. https://doi.org/10.1007/s10995-0121194-5.

Woodley XM, Lockard M (2016). Womanism and snowball sampling: Engaging marginalized populations in holistic research. The Qualitative Report. 21(2): 321-329. https://nsuworks.nova.edu/tqr/vol21/iss2/9/. 